favorables comienzan a naturalizarse.

Tragopogon angustifolius Bellardi ex Willd.

* MURCIA: Cartagena, Cabezo de las Colmenas 30SXG8762, $160 \mathrm{~m}$, pastizales nitrificados. J. F. Jiménez \& M. A. Carrión Vilches. 26-IV-1998. MGC48198. Taxon frecuentemente confundido o asimilado a Tragopogon crocifolius $\mathrm{L}$.

\section{BIBLIOGRAFÍA}

ESTEVE, F. -1973-Vegetación y flora de las regiones central y meridional de la provincia de Murcia. Publ. Diputac. Murcia-C.S.I.C.

PALLARÉS NAVARRO, A. -1999- Orquídeas de Almería. Almería, 238 pp.

SÁNCHEZ-GÓMEZ, P., J. GUERRA, E. COY, A. HERNÁNDEZ, S. FERNÁNDEZ y A. F. CARRILLO -1998a- Flora de Murcia. Claves de identificación e iconografía de plantas vasculares. DM Ed. Lérida, 439 pp.
SÁNCHEZ-GÓMEZ, P., J. GUERRA, J. GÜEMES, J. GARCÍA, A. HERNÁNDEZ, A. F. CARRILLO \& M. A. CARRIÓN -1998b- Flora Murciana de Interés Nacional y Europeo. Protección y Legislación. Universidad de Murcia. Consejería de Medio Ambiente, Agricultura y Agua de la Región de Murcia. Fundación SÉNECA. Murcia, 70 pp.

TALAVERA, S. y M. ACOSTA -1998- Notas sobre el género Colutea (Leguminosae) en España. Anales Jard. Bot. Madrid 56 (2):410-416.

Aceptado para su publicación en Octubre de 1999

Dirección de los autores. Departamento de Biología Vegetal (Botánica). Facultad de Biología. Campus de Espinardo. 30100. Murcia. E-mail: psgomez@fcu.um.es

\title{
90. UNA SUBESPECIE NUEVA EN EL GÉNERO SIDERITIS L. (LAMIACEAE)
}

\author{
Manuel Benito CRESPO VILLALBA, José Luís SOLANAS FERRÁNDIZ, \\ Antonio DE LA TORRE GARCÍA y Joaquín PAYÁ MIRA
}

A new subspecies in the genus Sideritis L. (Lamiaceae).

Palabras clave. Sideritis, Lamiaceae, Alicante, España.

Key words. Sideritis, Lamiaceae, Alicante, Spain.

Se propone una nueva subespecie, Sideritis chamaedryfolia Cav. subsp. littoralis M.B. Crespo, Solanas, De la Torre et Payá, después de estudiar el material existente en el
Herbario de la Universidad de Alicante (ABH) recogido en los matorrales sabulícolas de la Sierra Helada ( Alfaz del Pí, Alicante). 
Sideritis chamaedryfolia Cav. subsp. littoralis M.B. Crespo, Solanas, De la Torre et Payá, subsp. nova

A subsp. chamaedryfolia typica differt habitu prostrato vel procumbente; caulibus goniotrichis; foliis bracteisque dentatospinosis; calyce campanulato, dentibus acuminatis; corollis minoribus, labio superiore breviter bilobato.

Holotypus: Hs, ALICANTE, Alfaz del Pí (Sierra Helada), 30SYH5571, $150 \mathrm{~m}$, in collibus aridis arenosis juxta maris, 5-IV-1998, A. Ruiz de León, J. Llull et J.C. Cristobal legerunt (ABH 40271).

Isotypus: ibidem (ABH 43469)

Ic: Fig. 1.

Caméfito (1-3 dm), con tallos arqueadoascendentes. Tallo y eje de la inflorescencia goniótricos, con glándulas abundantes y sin pelos glandulares, indumento hirsutoide formado por pelos arqueados antrorsos 0 patentes, menos abundantes y más cortos que en la subespecie tipo. Hojas 10-20 x 4-5 mm, sentadas, pinnato-lobuladas, obovadas, en general con 2-3 pares de dientes bien marcados y terminados en espina robusta, con escasas glándulas y sin pelos glandulares. Haz glabro y envés glabrescente con pelos aislados en la nervadura. Inflorescencia en verticilastros, con 4-6 flores por verticilastro. Brácteas 5-6 x 10$12 \mathrm{~mm}$, más cortas que el cáliz, anchamente ovales, con 3-4 dientes a cada lado, más finos y alargados que los de la subespecie tipo, con aspecto espinoso, con abundantes glándulas y escasos pelos que se encuentran alrededor de la base de la bráctea. Cáliz 8-9 mm, campanulado, con 5 dientes subiguales de 4 $\mathrm{mm}$, con aspecto espinoso, con abundantes glándulas y escasos tricomas repartidos homogéneamente por toda la superficie externa, sin carpostegio o este discontínuo. Corola 7-8 mm, cubierta de pelos finos patentes, bilabiada, con labio superior de longitud $1 / 2$ la del inferior, ligeramente bífido, amarillo, el inferior con tres lóbulos desiguales y amarillo. Florece de marzo a junio.

Habita exclusivamente en los matorrales litorales propios de suelos de textura gruesa de la Sierra Helada (Benidorm y Alfaz del Pí), en el piso termomediterráneo semiárido (RivasMartínez, 1999). Ecológicamente, se desarrolla sobre arenosoles calcáricos, como la subsp. chamaedryfolia pero la subsp. littoralis lo hace bajo condiciones climáticas mucho más térmicas y oceánicas, debido fundamentalmente a la proximidad de la costa.

Morfológicamente se diferencia de la subespecie tipo por su hábito prostradoprocumbente y porque tanto las brácteas como el cáliz desarrollan dientes más marcados, llegando a tener incluso aspecto espinoso. En cuanto a la corola, en este caso es ligeramente más pequeña con el labio superior brevemente bífido. Además, presenta el eje de la inflorescencia goniótrico, a diferencia de la subespecie típica que lo tiene holótrico, rasgo que también se manifiesta en Sideritis dianica Rivera et al., especie de distribución próxima, lo que induce a pensar que el nuevo táxon pudo tener su origen por introgresión entre esta especie y la subsp. chamaedryfolia. Por el contrario, Sideritis dianica difiere de la nueva subespecie, entre otros caracteres, porque presenta el indumento del cáliz más largamente hirsuto, hojas más lobuladas y por su preferencia no sabulícola.

Sideritis chamaedryfolia subsp. littoralis es un endemismo que ocupa una superficie muy reducida en las dunas colgadas de Sierra Helada, entre Benidorm y Alfaz del Pí, y que caracteriza los matorrales de arenas litorales del límite septentrional del subsector Alicantino (Alcaraz et al., 1991) representados por la asociación Teucrio dunensisThymelaeetum valentinae Solanas (1996). Además, la presencia en este ecosistema de otras especies de elevado valor ecológico como Helianthemum violaceum var, arabinianum, Linaria arabiniana, Biscutella rosularis, 

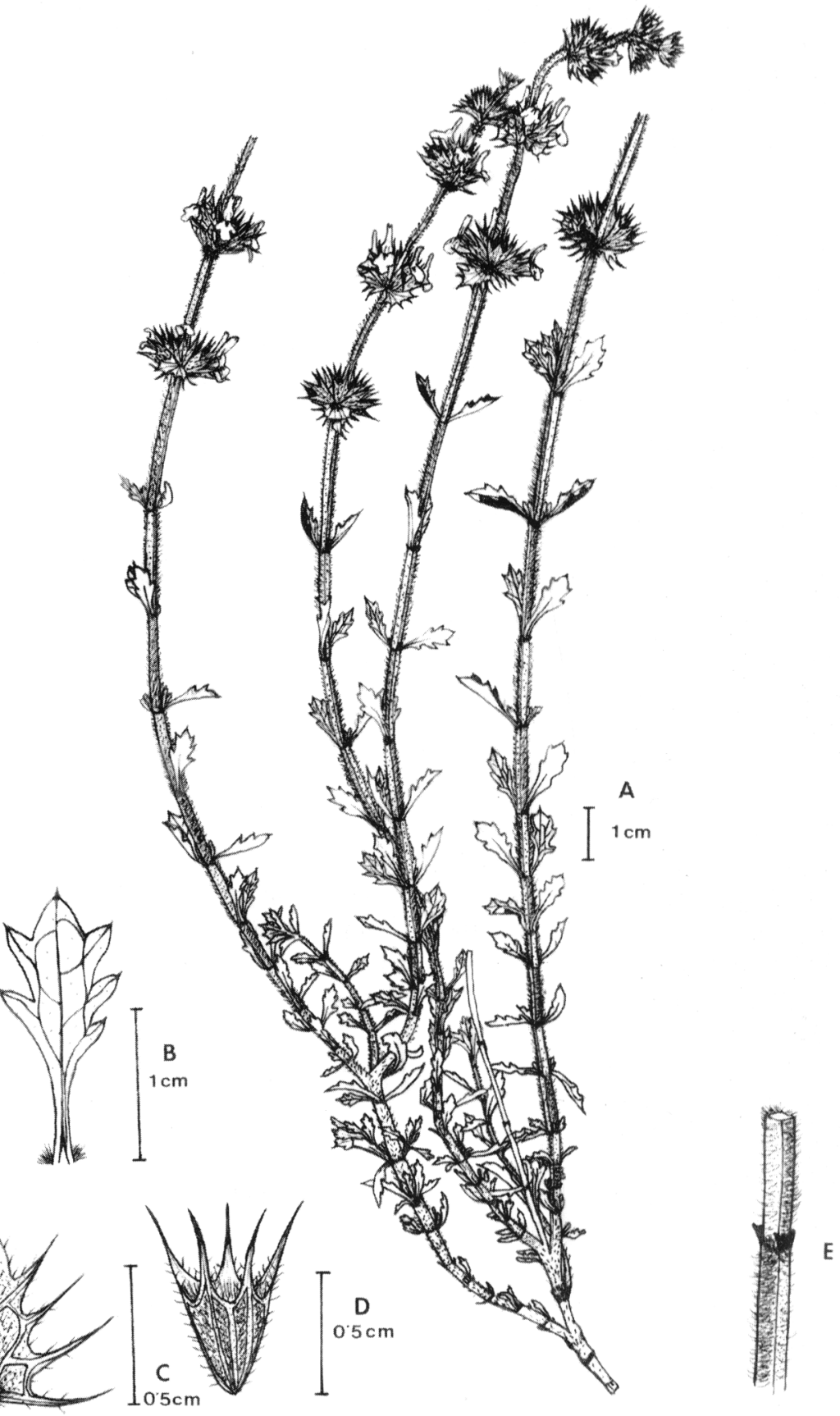

Figura 1. Sideritis chamaedryfolia subsp. littoralis: A, hábito; B, hoja; C, bráctea; D, cáliz; E, fragmento del tallo. Sideritis chamaedryfolia subsp. littoralis: A, habit; B, leaf; $C$, bract; $D$, calyx; E, stem fragment. 
también características de estos matorrales, y Juniperus macrocarpa, Corema album, Chamaerops humilis, etc., especies que conforman la comunidad clímax en estas arenas (Coremo albi-Juniperetum macrocarpae Alcaraz et al. (Solanas, 1996)), ha llevado a incluir a las playas o dunas colgadas de Sierra Helada en el listado de hábitats de la Directiva 92/43/CEE para su conservación. En este sentido, a nivel autonómico, se ha incluido esta zona en la red de microrreservas de flora de la Comunidad Valenciana, cuya finalidad es destacar o conservar las especies vegetales endémicas, raras o amenazadas y las formas singulares de vegetación natural que en las microrreservas se desarrollan (Laguna et al., 1998).

Actualmente, estos ecosistemas dunares se conservan en buen estado fundamentalmente por su inaccesibilidad, pero debido a la gran especulación urbanística en las proximidades, derivada del turismo, deberían tomarse medidas que garantizasen la conservación de estos ambientes dunares en el futuro.

\section{MATERIAL ESTUDIADO}

ALICANTE: Benidorm, Sierra Helada, YH5671, $50 \mathrm{~m}$, M.B. Crespo, A. de la Torre et J.L. Solanas (ABH 350); Alfaz de Pi, Sierra Helada, 15 m, YH5671, A. Ruiz de León, J.C. Cristóbal et al. (ABH 38098); Alfaz del Pi, Sierra Helada, YH5671, $100 \mathrm{~m}$, A. Ruiz de León, L. Rull et J.C. Cristóbal (ABH 39079); Alfaz del Pi, Sierra Helada, YH5671, $20 \mathrm{~m}$, A. Ruiz de León et P. Malaxechevarría (ABH 39534); Alfaz del Pi, Sierra Helada, YH5571, 100 $\mathrm{m}$, A. Ruiz de León, L. Rull et J.C. Cristóbal (ABH 40279); HOLOTIPO: Alfaz del Pi, Sierra Helada, YH5571, 150 m, A. Ruiz de León, L. Rull et J.C. Cristóbal (ABH 40271).

\section{BIBLIOGRAFÍA}

ALCARAZ, F., P. SÁNCHEZ-GÓMEZ y A. DE LA TORRE -1991- Biogeografía de la provincia Murciano-Almeriense hasta el nivel de subsector. Rivasgodaya 6: 77-100.

LAGUNA, E., coord., -1998- Flora endémica, rara o amenazada de la Comunidad Valenciana, Ed. Generalitat Valenciana, Consellería de Medio Ambiente. Valencia, 443 pp.

RIVAS-MARTÍNEZ, S. -1999- North American boreal and western temperate forest vegetation (Syntaxonomical synopsis of the potential natural plant comunities of North America, II). Itinera Geobotánica 12: 5-316.

SOLANAS, J. L. -1996- Flora, vegetació y fitogeografia de la Marina Baixa. Tesis Doctoral inédita. Universidad de Alicante. Alicante, 732 pp.

Aceptado para su publicación en julio de 2000

Dirección de los autores. Departamento de Ciencias Ambientales y Recursos Naturales. Universidad de Alicante. Apartado 99. E-03080 Alicante. 